\title{
Intellectual disability-alacrima-achalasia syndrome
}

INSERM

\section{Source}

INSERM. (1999). Orphanet: an online rare disease and orphan drug data base. Intellectual disability-alacrima-achalasia syndrome. ORPHA:289483

Intellectual disability-alacrima-achalasia syndrome is a rare, genetic intellectual disability syndrome characterized by delayed motor and cognitive development, absence or severe delay in speech development, intellectual disability, and alacrima.

Achalasia/dysphagia and mild autonomic dysfunction (i.e. anisocoria) have also been reported in some patients. The phenotype is similar to the one observed in autosomal recessive Triple A syndrome, but differs by the presence of intellectual disability in all affected individuals. 